\title{
Slant judgments from static and rotating trapezoids correspond to rules of perspective geometry*
}

\author{
RICHARD K. OLSON \\ University of Colorado, Boulder, Colorado 80302
}

\begin{abstract}
Apparent static slant of trapezoids aligned in the observer's frontal plane was compared with the apparent plane of reversal for the same trapezoids when rotated. Slant responses were similar in both static and rotation conditions, thus allowing description of the trapezoidal window illusion in terms of projective geometry. Linear perspective was systematically varied in the stimulus series to geometrically imply slants of $50,60,70$, and $80 \mathrm{deg}$. Perceived slant corresponded remarkably well with these values. The results show that with the proper viewing conditions and response measures, observers may be rather good perspective geometers.
\end{abstract}

The trapezoidal window illusion has been the subject of some theoretical interest and much fascination since Ames (1951) introduced it. Typically, the $S$ viewed the slowly rotating trapezoid with one eye at a distance of about $10 \mathrm{ft}$. Ames (1951) described the resulting illusion as follows: "As the trapezoidal window slowly rotates about a vertical axis, instead of appearing to rotate completely around, it appears to oscillate back and forth through an angle of about $100^{\circ}$ [p. 15]." The trapezoidal window illusion is but one example of a larger class of important perceptual phenomena in which the usual depth information (binocular and motion parallax) is reduced or absent. This study attempts to isolate some of the variables contributing both to slant perception from trapezoidal stimuli and the illusion of oscillation.

The stimulus information of interest is the trapezoidal contour outline. How do variations in outline shape of a trapezoid affect the observer's perception of depth (slant) and illusory oscillation? Suppose that, under certain viewing conditions, the observer tends to see a trapezoidal projection on the retina as a slanted rectangle. By rules of projective geometry (see Braunstein \& Payne, 1969), a trapezoidal retinal projection may be cast by a rectangle at a specified slant to the line of sight. How good a projective geometer is the $S$ ? Given the assumption that the trapezoidal retinal projection is caused by a slanted rectangle, how closely will the S's perceived slant be related to geometrically implied slant? Also, when a trapezoid shape is rotated, how will the projective geometry affect the illusion of oscillation?

The Ames trapezoidal window illusion, with its motion component, may seem to be of a separate class, but the principles alluded to above for static trapezoidal

\footnotetext{
*This research was supported by the Advanced Research Projects Agency of the Department of Defense, monitored by the Air Force Office of Scientific Research under Contract No. F44620-67-C-0099, and by NIMH Research Grant MH20449 to Fred Attneave. The author thanks Fred Attneave for his assistance. This study was the author's PhD dissertation.
}

retinal images may also be applied to perceived oscillation of rotating trapezoids. A major goal of this study is to discover if the $S$ responds to the trapezoidal window illusion in a fashion consistent with, or at least related to, contour outline and the rules of projective geometry.

Most previous accounts of the rotating trapezoid illusion simply pointed to the fact that the long end of the trapezoid is seen as closer to the $\mathrm{S}$ than the short end (Ames, 1951; Borjesson, 1971; Graham, 1963; Graham \& Gillam, 1970; Power \& Day, 1973). See Graham (1963) for a diagramatic account of the illusion. The geometric implications of trapezoid shape and the particular angle of apparent plane of reversal were not considered. Apparent reversal occurs approximately when the rotating trapezoid passes the S's frontal plane. Ames suggested that the perceived angle of oscillation was about $100 \mathrm{deg}$ in his observations, although no precise measurements were made and there was no attempt to relate this to stimulus variables.

The present study directly compared perceived angle of oscillation and variations in trapezoid shape by having Ss estimate apparent slant at the two planes of reversal. These judgments were compared with static slant judgments of identical trapezoidal stimuli aligned in the S's frontal plane.

\section{METHOD}

\section{Subjects}

The nine $\mathrm{Ss}$, three men and six women, were paid undergraduate volunteers from the University of Oregon.

\section{Stimuli}

The set of trapezoidal stimuli represented in Fig. 1 requires a somewhat detailed explanation. The major stimulus variable of interest is slant implied by linear perspective. Trapezoids were constructed to cast the same retinal images as rectangles slanted $50,60,70$, or $80 \mathrm{deg}$ from the S's frontal plane when the trapezoids were oriented in the S's frontal plane at a viewing distance of $2.74 \mathrm{~m}$. These levels of implied slant correspond to the four columns of Fig. 1.

Within each column of Fig. 1, there are five trapezoids 


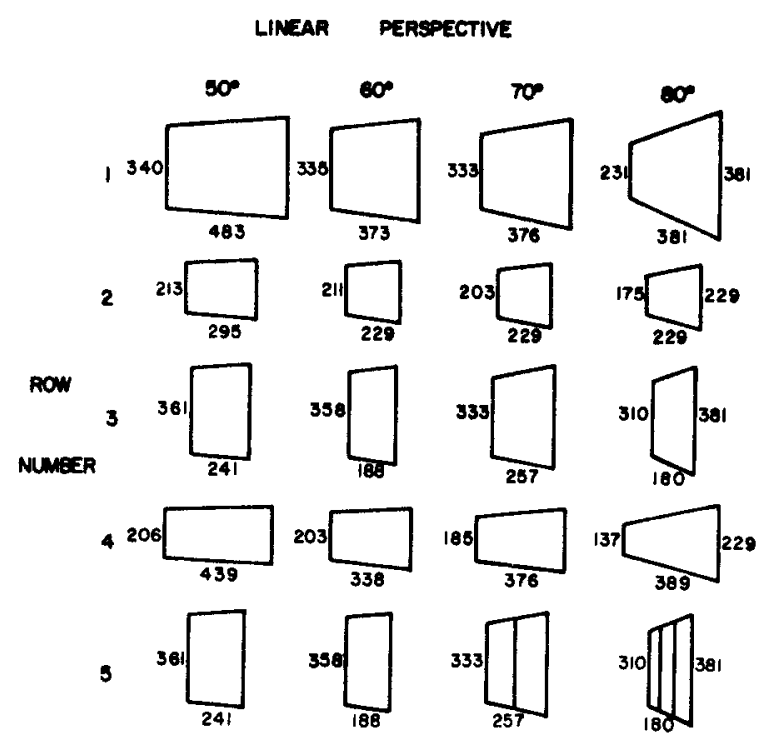

Fig. 1. Height of small end and width of trapezoids are given to the nearest millimeter, respectively, to the left and below each stimulus. Heights along the vertical midline are either 229 or $381 \mathrm{~mm}$, as noted in the right margin. Rows 1-4 refer to the four main levels of height-width variation. Row 5 includes stimuli designated as "squares" or "divided into squares," as this would be true of the implied slanted rectangle.

representing the same slant (as implied by linear perspective) of rectangles that differ, however, in shape and size. Trapezoids in Rows $1-4$ of each column vary in height along their vertical midline (229 or $381 \mathrm{~mm}$ ). Deviations from the exact 229- and $381-\mathrm{mm}$ width values will be explained in the next several paragraphs. Variations in height and width were introduced primarily to provide differences in shape other than those directly related to geometrically implied slant. This was to avoid any tendencies $S$ might have to artificially order his slant responses on a single stimulus variable, such as angle of trapezoid side convergence.

There are several important consequences of height-width variation. Trapezoids from Rows 1 and 2, Fig. 1 , differ in midline height and width, but havy nearly the same ratio of height to width, about 1 to 1 . In this respect, they differ in size, not shape. However, angle of convergence or size of the angle between the sides is different between Rows 1 and 2 . One investigation of shape-slant relationships has suggested that angle of convergence per se may be the appropriate stimulus variable for perceived slant (Freeman, 1966). Evaluation of the S's responses to stimuli in Rows 1 and 2 should indicate whether they are responding to angle of convergence per se or geometrically implied slant.

Another result of height-width variation is the difference in height-width ratio (HWR) of the frontal trapezoids. As already noted, stimuli in Rows 1 and 2 have nearly the same HWR within each level of implied slant. It is approximately 1 to 1 . Stimuli in Row 3 have a HWR greater than 1 , about 15 to 9 . HWR of stimuli in Row 4 is less than 1, approximately 9 to 15 .

HWR may also be considered with respect to the dimensions of the implied rectangle. Herein lies the rationale for stimuli in Row 5 and variations about 229- and $381-\mathrm{mm}$ width values in the whole stimulus matrix. Each of the stimuli geometrically imply (cast the same retinal image as) either slanted squares or slanted rectangles that may be evenly divided into squares. Since the height of trapezoids along the vertical midline was fixed at either 229 or $381 \mathrm{~mm}$, trapezoid length had to vary somewhat about these values to imply a square or rectangle divisible into squares. Only those trapezoids in Row 5 were actually divided into implied squares. They are identical in outline shape to those of Row 3, except that the appropriate implied square divisions were made in the 70- and 80-deg trapezoids. Fif ty-degree and 60-deg trapezoids imply single squares. When stimuli of Row 5 were presented, Ss were told they were squares or rectangles divided into squares. The question was, what effect would this assumption regarding implied rectangle HWR have on perceived slant?

All trapezoids of Fig. 1 were constructed to the specified dimensions with $1.59-\mathrm{mm}$ steel welding rod and painted fluorescent orange (Orr-lac No. 991). A 1.59-mm black painted rod was attached at the midline to the bottom side of each trapezoid to facilitate mounting for static display and rotation. For full-cue control and demonstration purposes, four rectangles were constructed to give projections nearly identical to the trapezoids of Fig. 1, Row 2, when set at the respective levels of implied slant. For example, length required of the 80-deg slanted rectangle was $1.37 \mathrm{~m}$ to match the 80 -deg trapezoid projection. Additional rectangles were constructed to project a $229-\mathrm{mm}$ wid th when set at $40,30,20$, and $10 \mathrm{deg}$ from the frontal plane. Eight training rods were constructed to familiarize $S$ initially with the response apparatus without training him on shape variables. These rods (and the above-mentioned rectangles) were made of the same painted welding rod as the trapezoids. The rods were cut to lengths which cast a visual angle projection of $4 \mathrm{deg} 40 \mathrm{~min}$ for slant angles 10 to $80 \mathrm{deg}$ in 10-deg steps at a viewing distance of $1.02 \mathrm{~m}$.

\section{Apparatus}

The viewing apparatus was designed to allow $S$ the full use of binocular vision in setting a response rod to the same apparent slant as that of the monocularly presented trapezoid. A view of the apparatus from above is shown in Fig. 2. A half-silvered mirror, tilted 45-deg to the S's line of sight, allowed superimposition of two viewing fields into the same virtual space. The reflected field was viewed binocularly. Monocular viewing of the nonreflected field was achieved with two Polaroid filters in the eyepiece with polarizing axes aligned orthogonally at $45 \mathrm{deg}$, and a large reversible filter in front of the stimuli. Monocular viewing of thestimulus by either the right or left eye could be achieved by changing the alignment of the large filter. Also, the large filter could be removed for binocular views of both fields.

The reflected response field contained an internally illuminated, neon-filled glass response rod, $457 \mathrm{~mm}$ long and $6.35 \mathrm{~mm}$ in diam, mounted horizontally at eye level through a vertical 12.7-mm black painted steel tube. Virtual distance from the S's eye to the middle of the response rod was $1.02 \mathrm{~m}$. The neon rod was masked except for a $3.17-\mathrm{mm}$-wide yellow strip the length of the rod on both sides. This strip was marked every $25 \mathrm{~mm}$ by $3.17-\mathrm{mm}$ sections of black tape. The yellow strip and inch marks were the only visible contours in the response field. Rotation of the response rod in the horizontal plane was accomplished by a pulley arrangement between the S's response wheel beneath the viewing box and a wheel of the same size on the vertical steel tube. The $E$ read angle of rod slant from a protractor attached to the top of the tube. A mask aperture, $63.5 \mathrm{~mm}$ wide, was placed $241 \mathrm{~mm}$ in front of the rod center to hold rod visual angle constant for both eyes up to 70 deg of slant from the frontal plane. This assured that Ss would not simply match visual angle extents of stimulus and rod.

Trapezoids and rectangles were shown in the nonreflected stimulus field at eye level, $2.74 \mathrm{~m}$ from the $\mathrm{S}$. Their central vertical axis was aligned with the reflected response rod to a point intermediate between the left and right eyes. Stimuli were mounted at their midline to a variable-speed motor drive socket arrangement by $1.59-\mathrm{mm}$ black painted rods. Speed in the rotation condition was $6 \mathrm{rpm}$. Training rods were supported by a stand slightly below eye level, $1.02 \mathrm{~m}$ from the $S$. The stimulus room was painted and flocked black. When an overhead 
fluorescent lamp was turned off, the stimuli were illuminated by two GE FGTS ultraviolet lamps; no extraneous contours were visible. A wall separated $S$ from the stimulus room.

This apparatus is similar in principle to that of Attneave and Frost (1969). Its major advantage is the compatibility and close association of stimulus and response. Ss find the task of relating apparent orientation of the monocular stimulus and the binocular response rod quite natural af ter a brief training period.

\section{Procedure}

Each $S$ was run for a total of 12 days, approximately $1 \mathrm{~h}$ per day. Major parts of the procedure included training with response rod, instructions, and initial practice with trapezoids on Day 1. Experimental trials with trapezoid stimuli were presented on Days 2-9. Three control conditions and a final question session were given on Days 10-12.

First, $\mathrm{S}$ learned to set the response rod parallel to training rods seen in full binocular vision with stimulus room lights on and Polaroid filter removed. Two trials, one with near end of the training rod to the left and one to the right, were given at each of the eight slant levels, 10 to $80 \mathrm{deg}$ in 10-deg steps from the frontal plane. All Ss were easily able to match orientation of the rods with less than 10-deg error. S was asked to begin each response with several wide sweeps of the rod, and then to gradually settle on the final orientation. This minimized effects of previous settings and helped to maintain depth perspective on the response rod.

Following rod practice, $S$ set his response rod parallel to each of four rectangles, casting $229 \times 229 \mathrm{~mm}$ projections at slants of $20,40,60$, and $80 \mathrm{deg}$ at the $2.74-\mathrm{m}$ viewing distance. They were viewed under full binocular vision with room lights on. No feedback was given, since the purposes of these four trials was to help establish S's "stimulus attitude," and it was not to train him in responding to projective shape variables. "Stimulus attitude" refers to S's assumption, supported by E's demonstrations and instructions, that stimuli were slanted and oscillating rectangles of various dimensions. The oscillation phenomenon with its left and right planes of reversal was demonstrated with an oscillating rectangle under full-cue conditions. $S$ was then told he would be viewing oscillating and stable rectangles of various sizes and angles of slant under reduced-cue conditions. The $E$ allowed that oscillating rectangles might occasionally seem to be rotating. In this case, $S$ was told to wait and make his response only when the stimulus was perceived as oscillating. Occurrences of rotation were to be verbally reported after the slant response. E emphasized that responses should be "natural," not figured out. The S was told that whatever slant seemed right to him was the desired response. No feedback was given.

Following training rod practice and instructions, each of the 60-, 70-, and 80-deg trapezoids in Fig. 1 was presented once in a practice series to familiarize $S$ with experimental procedures and variables. Stimulus order, viewing eye, plane of reversal or slant, and the rotation vs stable conditions were randomly selected for each trial. In a typical rotation trial, $E$ announced the plane of reversal to be set ("near end" of the apparent rectangle to the left or right), started the rotation motor, and opened a shutter on S's viewer. The S made his response without time restriction and then reported any perceived rotation. In the stationary condition, $E$ aligned the trapezoid in $S$ 's frontal plane, long side left or right. The occurrence of "square" stimuli (Fig. 1, Row 5) was announced before those trials: "This stimulus is a square" or "This stimulus is divided in to squares." Stationary slant and apparent plane of reversal (APR) responses were recorded in degrees of slant from the frontal plane by $E$ at the end of each trial.

The complete set of 20 trapezoids was presented in random order in each of eight experimental sessions. Rotation vs stable and left vs right plane of reversal or slant conditions were randomly selected for a given stimulus in the first of two experimental sessions and reversed in the second. Viewing eye

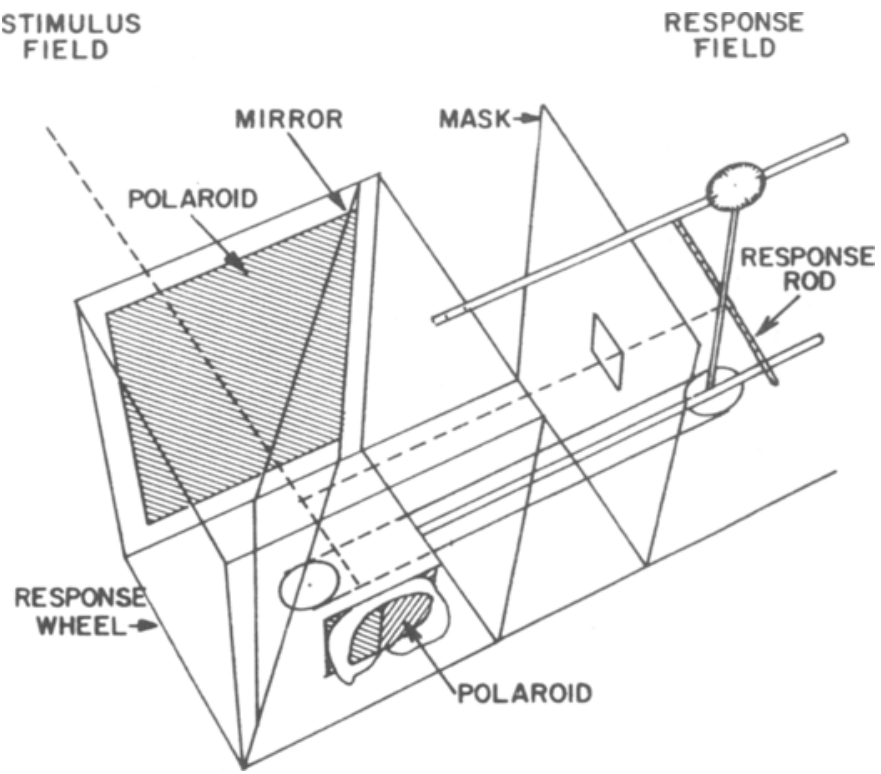

Fig. 2. The response apparatus.

was alternated every two series. Direction of rotation was counterbalanced over Ss.

On Day 10, trapezoids in Fig. 1, Row 2, were viewed only in the stationary frontal orientation to control for possible effects caused by the close temporal contiguity of rotating and stationary trials. The 16 possible combinations of stimulus, right or left viewing eye, and right or left plane of slant were randomly selected with elimination on each trial. The same procedure and stimuli were given on Day 11 under the rotation condition.

In the second half of the hour on Day 11 , Ss were asked to stop the stimuli at the APR with a hand switch. The response rod was darkened, and no explicit slant responses were required. The Ss simply stopped the motor when the assumed rectangle reached its APR. Three trapezoids employed in this condition were those in Fig. 1, Row 4, for 60,70 , and $80 \mathrm{deg}$ implied stant. Stimulus, direction of rotation, and left-right APR combinations were randomly selected with exclusion on each trial. Two of the above series were presented, one for the left and one for the right eye. E read stopped orientation of the trapezoids from a protractor beneath the stimulus.

On Day 12, eight full-cue rectangles were shown at randomly selected 10-deg-separated levels of slant, 10 to $80 \mathrm{deg}$. Further randomization over left-right plane of slant and two replications yielded a total of 32 trials. At the end of the experiment, Ss were questioned about their experiences and attitudes during the experimental trials. Then they were taken into the stimulus room and shown the trapezoidal stimuli.

\section{RESULTS AND DISCUSSION}

One $\mathbf{S}$ was unable to perceive oscillation with some of the stimuli and was dropped from the experiment. The other eight Ss completed all experimental and control conditions. Each S's performance in the experimental tasks was first examined as a function of right vs left viewing eye, right vs left APR, and right vs left static plane of slant. No consistent effects of these variables were noted over Ss. Thus, each S's responses to trapezoids in the main experimental series were averaged 


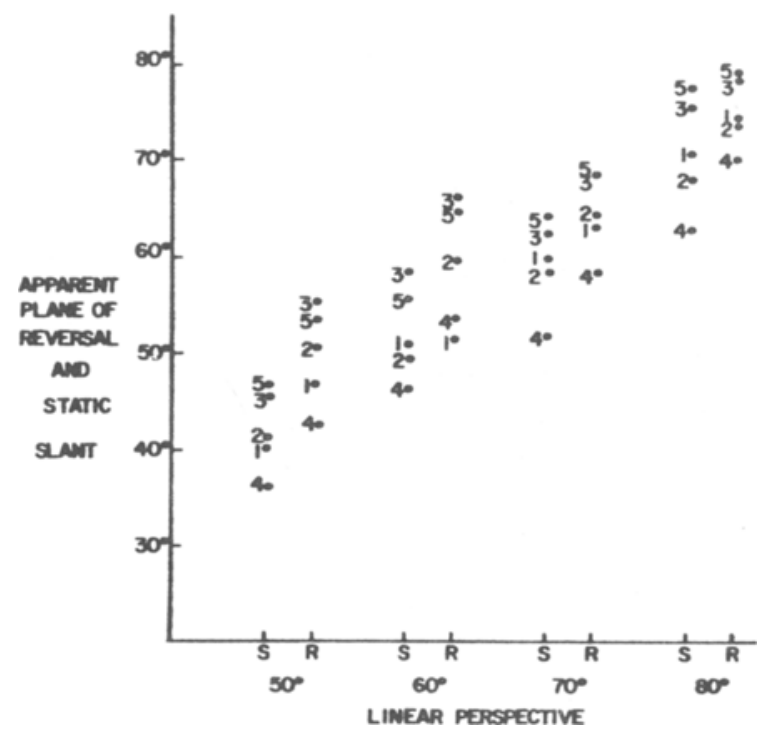

Fig. 3. Plotted numbers refer to stimulus row number in Fig. 1. $S$ and $R$ refer to stationary and rotating conditions, respectively.

over left-right viewing eye and left-right APR or slant, yielding two means for each stimulus, one for stationary slant and one for APR. Ss' performances were sufficiently similar to justify averaging their means, yielding an APR and static slant grand mean for each stimulus. Means so obtained are plotted in Fig. 3.

The data were evaluated in a three-way repeated-measures analysis of variance from Myers, 1966). Results of the analysis are summarized in Table 1. Notation and procedure for Tukey's test of the significance of differences between cell means referred to in the following paragraphs was taken from Guenther (1964).

As can be seen in Fig. 3, APR of rotating and perceived slant of stationary trapezoids revealed remarkably similar trends. However, the analysis of variance indicated some differences. Most obvious from Fig. 3 is the main effect of rotation ( $\overline{\mathrm{X}} \mathrm{APR}=$ $62.18 \mathrm{deg})$ vs stationary ( $\overline{\mathrm{X}}$ slant $=56.10 \mathrm{deg}$ ) viewing conditions. Although this difference was statistically significant (see Table 1), differences over individual Ss were relatively inconsistent. Three Ss produced mean differences of less than $1 \mathrm{deg}$, but differences for the other five Ss ranged from about 5 to $15 \mathrm{deg}$, averaging $9.5 \mathrm{deg}$. For these five $\mathrm{Ss}$, the difference between rotating and stationary conditions amounted to a general shift in absolute level of slant response similar to that shown in Fig. 3.

Marginally significant interactions of rotation vs static slant (A) with linear perspective (B) and height-width variation (C) (see Table 1) indicate that the shift was not completely even. These interactions are plotted in Fig. 4. The A by B interaction suggests that the magnitude of the shift was slightly greater for.lower levels of implied slant. The A by C interaction shows some minor differences in responses between rotation and static viewing as a function of height-width variation. The author attributes no particular theoretical significance to these interactions.

Results from two control conditions on Days 10 and 11 indicate that the basic similarity of performance was not due to close temporal proximity of rotation and static responses in the experimental series. Mean perceived slant for frontally aligned $229 \times 229 \mathrm{~mm}$ trapezoids presented on Day 10 was $57.1 \mathrm{deg}$. In the experimental trials, stationary responses to these same stimuli averaged $54.2 \mathrm{deg}$. This difference is not statistically significant $(t=1.50 ; \mathrm{df}=1,7 ; \mathrm{p}>.1)$. Mean APR for these stimuli on Day 11 in the rotation condition was $58.5 \mathrm{deg}$ compared to $62.1 \mathrm{deg}$ in experimental trials $(t=1.23 ; d f=1,7 ; p>.1)$. Thus, similarity of performance is maintained with a 1-day temporal separation between stationary and rotation conditions.

A possible basis for the similarity of apparent plane of reversal and static response is suggested by Ss' attempts to stop the trapezoids at their APR on Day 11. Most responses were less than $5 \mathrm{deg}$ from the frontal plane. Thus, the retinal images at the APR were nearly identical to the static slant condition. Why, then, did five of the eight Ss give slightly more slanted responses to the rotating stimuli? Perhaps some Ss found slant a more salient aspect of the moving stimulus, as other studies have found with the "kinetic depth effect" (Gibson \& Gibson, 1957; Wallach \& O'Connell, 1953; Wallach, O'Connell, \& Neisser, 1953). A plausible alternative is that some Ss tend to compromise their APR responses toward the greater slants perceived at other stages of rotation. Note that the APR response is a judgment of the minimum slant that occurs during oscillation, that the duration of this minimum is infinitesimal, and that

Table 1

Analysis of Variance Treatment of Subjects' Mean Static Slant and Apparent Plane of Reversal Responses to Trapezoids

\begin{tabular}{lrrrr}
\hline \multicolumn{1}{c}{ Source } & df & $\begin{array}{c}\text { Mean } \\
\text { Squares }\end{array}$ & $\begin{array}{c}\text { F } \\
\text { Value }\end{array}$ & $\begin{array}{c}\mathrm{p} \\
\text { Value }\end{array}$ \\
\hline Within & & & & \\
Rotation vs Static (A) & 1 & 2958.15 & 8.43 & $\mathrm{p}<.025$ \\
A by Subjects (S) & 7 & 350.90 & & \\
Linear Perspective (B) & 3 & 10661.46 & 80.22 & $\mathrm{p}<.001$ \\
B by S & 21 & 132.90 & & \\
Height-Width & 4 & 1431.21 & 15.74 & $\mathrm{p}<.001$ \\
Variation (C) & & & & \\
C by S & 28 & 90.90 & & \\
A by B & 3 & 54.62 & 3.99 & $\mathrm{p}<.05$ \\
A by B by S & 21 & 13.70 & & \\
A by C & 4 & 62.01 & 3.71 & $\mathrm{p}<.05$ \\
A by C by S & 28 & 16.73 & & \\
B by C & 12 & 23.36 & 1.73 & n.s.* \\
B by C by S & 84 & 13.51 & & \\
A by B by C & 12 & 17.52 & .68 & n.s. \\
A by B by C by S & 84 & 25.79 & & \\
\hline
\end{tabular}

*Not significant at $\alpha=.05$ 
any averaging of apparent slants in the minimum region would necessarily inflate the value of the slant response.

\section{Effects of Linear Perspective}

Stimulus differences in linear perspective (geometrically implied slant) had a very strong effect on the Ss' slant responses. Mean responses were 45.8, 55.6, 62.0 , and $73.3 \mathrm{deg}$ when averaged over height-width variation and rotation vs static viewing conditions. These results were surprisingly close to geometrically predicted slants of $50,60,70$, and $80 \mathrm{deg}$, respectively.

Whether the above results represent true perceived slant or some slant bias associated with the response rod was evaluated in the full-cue (room lights on and binocular viewing) rectangle control condition. Mean responses to the eight slanted rectangles were $12.4,25.0$, $34.9,44.7,53.4,63.5,72.3$, and $80.1 \mathrm{deg}$. Thus, response rod settings to full-cue stimuli were quite consistent with their actual slant. Apparent slant of trapezoids (in the Day 10 control condition) giving retinal images equivalent to 50-, 60-, 70-, and 80-deg full-cue rectangles was $40.1,51.0,59.9$, and $71.1 \mathrm{deg}$, respectively. The mean regression of perceived frontal trapezoid slant from perceived full-cue rectangle slant was $13.1 \mathrm{deg}(\mathrm{t}=5.63 ; \mathrm{df}=1,7 ; \mathrm{p}<.01)$. Factors contributing to this regression may involve more than the single fact that rectangles were viewed binocularly. Constant brightness and width of trapezoid contours may have competed with depth information from linear perspective. Also, accommodation may provide some information regarding actual depth of stimulus contours. A third possibility will be considered in the discussion of height-width ratio effects.

Ss in this study showed much closer correspondence of perceived static slant with perspective-implied slant than that found by other investigators (Gibson \& Gibson, 1957; Smith, 1964; Flock et al, 1967). One major difference between these earlier studies and the study reported here was the close temporal contiguity of rotating and static slant trials. In the earlier studies, the Ss viewed static stimuli exclusively. It thus might be suggested that the present static slant responses were somehow dependent on the intervening presentation of, and responses to, rotating stimuli. Recall, however, that a control study showed that $24 \mathrm{~h}$ separation between static and rotation trials did not diminish but, rather, increased the similarity of static and rotating responses.

The importance of an appropriate response measure should not be overlooked. Previous studies have used methods of estimating slant that were often awkward for the $S$ and that sometimes involved changing the retinal shape of the response object, so that true depth responses might have been bypassed. The slant response method used in this study had two important features. (1) Slant of the rod was judged primarily from binocular disparity rather than projective shape; and (2) the rod was in the same virtual space as the stimulus, allowing simultaneous viewing of both. Depth or slant of the
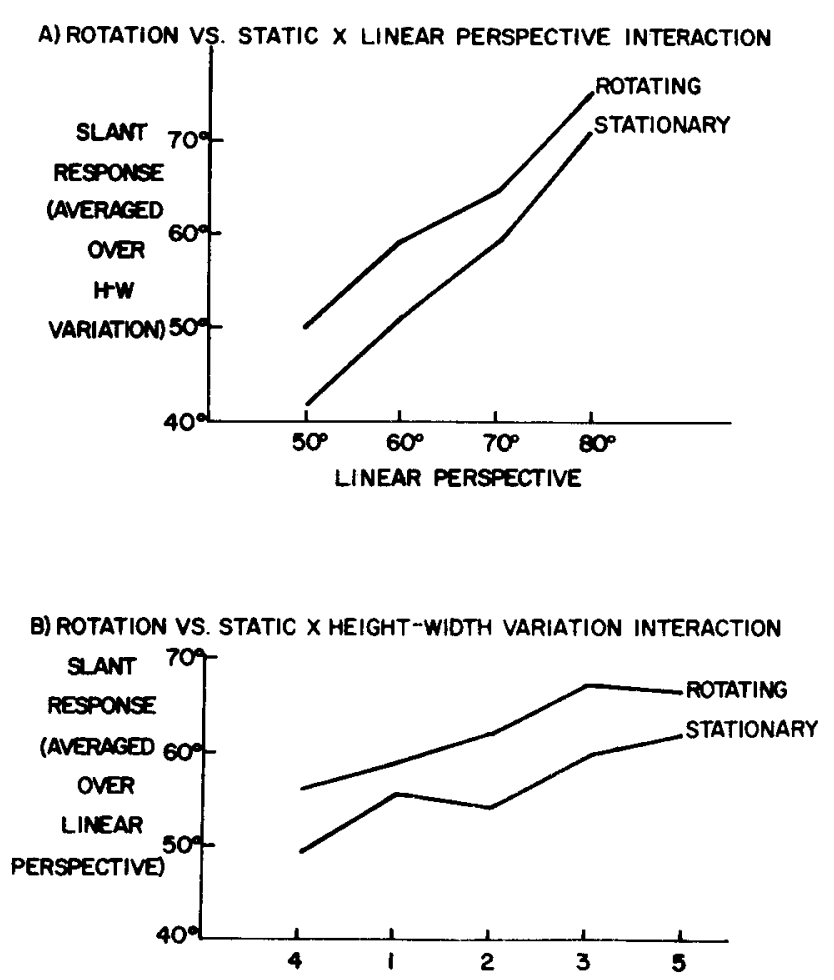

Fig. 4. Interactions of rotation vs static conditions with linear perspective and height-width variation.

response rod was perceptually quite obvious and could be matched directly with perceived slant of the stimulus.

Two other studies of apparent plane of reversal showed strong regression and little or no correspondence to perspective-implied slant (Freeman \& Pasnak, 1968; Epstein et al, 1968). This lack of correspondence to perspective predictions may have been due to their selection of stimuli with extreme geometrically implied slants and dimensions. Freeman and Pasnak (1968, Experiment 8) rotated four trapezoids, $254 \mathrm{~mm}$ long (horizontal extent) with long and short ends of 25.4 and $229 \mathrm{~mm}, 50.8$ and $203 \mathrm{~mm}, 76.2$ and $178 \mathrm{~mm}$, and 102 and $152 \mathrm{~mm}$, at a viewing distance of $2.74 \mathrm{~m}$. Analysis from projective geometry at the $2.74 \mathrm{~m}$ viewing distance for $25.4-$ and $229-\mathrm{mm}$ and $102-$ and $152-\mathrm{mm}$ frontal trapezoids gives implied slants from the frontal plane of about 88 and $77 \mathrm{deg}$, respectively. HWR of the implied rectangles would be about 1 to 77 and 1 to 7.7 , respectively. Epstein et al presented three trapezoids at $1.75 \mathrm{~m}$, each $114 \mathrm{~mm}$ wide and $114 \mathrm{~mm}$ long side. Sizes of the small angle were $67.6,75.4$, and $87.7 \mathrm{deg}$. These stimuli represent projections of rectangles slanted at $87 \mathrm{deg} 20 \mathrm{~min}, 84 \mathrm{deg} 40 \mathrm{~min}$, and $72 \mathrm{deg} 20 \mathrm{~min}$, respectively, from the frontal plane. Note that the range of geometrically implied slant is quite limited in both studies.

The Epstein et al Ss moved a crank to mimic the angle of oscillation. Mean angles of swing on the crank were 112,111 , and $113 \mathrm{deg}$. No effects of linear perspective 
were revealed. Freeman and Pasnak's Ss judged APR by positioning a pointer against a protractor below the eyepiece. Their mean APR judgments were approximately 40 and $35 \mathrm{deg}$, respectively, for the 88and 77-deg implied slants. Effects of linear perspective were said to be "highly significant." However, the results have little relevance to implied stimulus slant. Selection of a few stimuli implying extreme slants may have been responsible for the ambiguous results. Additionally, their response measures and the Ss' viewing attitudes may not have been conducive to geometrically appropriate spatial interpretations of the trapezoids. The Epstein et al Ss knew the stimuli were rotating trapezoids, while Freeman and Pasnak's Ss were left to draw their own conclusions.

With extreme stimuli and viewing situations less than optimal for interpreting slant from projectie shape, APR may be a highly variable compromise between geometrically implied slant and the frontal plane. In neither study was the APR even close to implied slant. It regressed at least half the angular distance to the frontal plane.

Freeman (1966) has suggested that angle of convergence of the two nonparallel sides is a stimulus for perceived slant. In their study of trapezoid oscillation, Freeman and Pasnak's (1968) confidence in this variable was indicated by their use of stimuli with a wide range of side angle convergence, but a relatively small range of geometrically implied slant.

In the study reported here, angle of convergence varies with trapezoid size, while geometrically implied slant is held constant with each column of Fig. 1. Compare the trapezoids in Rows 1 and 2 of Fig. 1 at each level of implied slant. The angles of side convergence are about $65 \%$ greater in Row 1 than in Row 2, while geometrically implied slants are the same between the rows. Does perceived slant follow the predictions of projective geometry or is it simply a function of angle convergence? The results unequivocally favor projective geometry. Mean slant and APR responses were nearly identical for the large and small trapezoids of Rows 1 and 2, 57.3 and 58.1 deg, respectively. On the other hand, a comparable difference in angle of convergence between 60 and $70 \mathrm{deg}$ implied slant columns of Fig. 1 yields a mean difference in perceived slant and APR of $6.45 \mathrm{deg}[\mathrm{p}(1.43 \leqslant \hat{\mathrm{L}} \leqslant$ $11.47)=.95]$. Linear perspective defined in terms of projective geometry seems to be the best predictor of slant responses to stimuli in this study. It would be desirable to replicate these findings with a wider range of height-width variation before drawing any final conclusions. Freeman's (1966) angle of convergence may be a more valid predictor of perceived slant with small visual angle (size on retina) stimuli.

\section{Effects of Height-Width Variation and "Square" Stimuli}

Height-width variation resulted in differences in the height-width ratio (HWR) of frontal trapezoids. In Rows
1 and 2 of Fig. 1, HWR is about 1 to 1. In Rows 3 and 4, HWR are about 15 to 9 and 9 to 15 , respectively. The effect of HWR on slant responses was statistically significant. Responses averaged over linear perspective and rotation vs static viewing for Rows 3 and 4 (the two extremes of trapezoid HWR) were 63.4 and $52.7 \mathrm{deg}$, respectively $[\mathrm{p}(5.79 \leqslant \hat{\mathrm{L}} \leqslant 15.61)=.95]$. The relationship between slant responses and HWR is shown in Fig. 3 for all trapezoids. Slant responses show a clear tendency to increase with an increase in HWR of the frontal trapezoid.

Interpretation of HWR effects in this study requires reference to some theoretical notions about space perception. Linear perspective provides the $S$ with geometrically valid information about surface slant. HWR of the retinal image may also provide valid information if information regarding the distal stimulus HWR is given to or assumed implicitly by the S. Epstein et al (1968) have used oscilloscope-presented ellipses to simulate oscillating circles. For some Ss, the perceived angle of oscillation was closely related to the slant of a circle required to cast the retinal image of the ellipse at its maximum width. Here, the only information about slant is HWR.

When stimuli in Row 5 of Fig. 1 were presented, the Ss were told that the stimuli were squares or made up of squares. Responses to Row 5 stimuli were not significantly different from those of Row 3 , where such specific information was not given for the same stimulus outlines. The mean slant responses were 63.4 and $64.2 \mathrm{deg}[\mathrm{p}(-4.08 \leqslant \hat{\mathrm{L}} \leqslant 5.708)=.95]$, respectively, for Rows 3 and 5 . The lack of a significant difference may have been due to the fact that slant responses to stimuli from Rows 3 and 5 were very close to perspective-implied slant. Also, in postexperiment questioning, Ss indicated that they did not respond to square stimuli in any special way.

The basis for Ss' responses to HWR in this study is not readily apparent. Except for Row 5, they were given no information about the assumed distal stimulus. In fact, they were told that they would see a large variety of rectangles. Perhaps the Ss tend to make a vague and unconscious perceptual inference that the distal stimulus has a HWR reasonably close to 1 . Such perceptual tendency might be based on learning and "unconscious inference" (Helmholtz, 1962) or a Gestalt-type preference for simplicity (Kofka, 1935; Pastore, 1952; Hochberg, 1964; Attneave, 1968). Extreme violations of a symmetry assumption, as would occur with stimuli in Row 4, might result in some regression in responses from slants implied by linear perspective. Attneave and Frost (1969) have found a similar sort of regression with different sources of two-dimensional depth information when one source suggests a frontal plane orientation.

\section{Stimulus Attitude and Rectangularity and Oscillation Assumptions \\ By means of instructions and demonstrations, the $\mathrm{E}$ attempted to establish the S's assumptions regarding}


rectangularity and oscillation of stimuli. Success in this attempt was mixed. One $\mathrm{S}$ was unable to perceive oscillation for most of the 50. and 60-deg linear perspective stimuli. However, APR responses to stimuli which this $\mathrm{S}$ could see as oscillating were similar to those of the other Ss. The eight Ss completing the experiment saw rotation during $17 \%$ of the experimental trials, but were able to perceive oscillation after a few moments. None of the 80-deg perspective stimuli were perceived to rotate. For the remaining stimuli, the number of perceived rotation trials was negatively correlated with mean perceived APR $(\mathrm{r}=-.926 ; \mathrm{t}=8.45 ; \mathrm{df}=12$; $\mathrm{p}<.001)$. Stimuli seen as rotating tended to be those with APRs closer to the frontal plane.

In postexperiment questioning, Ss indicated that they really believed the stimuli were oscillating when they set the response rod to the APR. However, six Ss said an effort of some sort was required to perceive oscillation with some of the stimuli. They could not specifically describe the nature of the effort. Three Ss mentioned that an overall view of the stimulus aided preception of oscillation. Burnham and Ono (1969) describe results suggesting that a "relaxed" view of the trapezoid is conducive to oscillation, while fixation on one of the ends of the trapezoid favors rotation.

Although a perceived rotating trapezoid should directly violate the rectangularity assumption, the Ss did not seem to be aware of this. When asked if any of the stimuli did not seem rectangular, three Ss thought they were all rectangles and the other five Ss indicated that the 80-deg perspective trapezoids, none of which were seen to rotate, looked somewhat nonrectangular. Perhaps if the Ss had been told to look for shape changes, as Burnham and Ono's Ss did, they would have become aware of the trapezoidal shape during perceived rotation. Apparently, the assumptions of rectangularity and oscillation were accepted by all but one $S$ for most of the stimuli. All Ss were astonished when they were shown the actual stimuli and rotation in the experimental room.

It is not clear how much the Ss' explicit assumptions of rectangularity and oscillation contributed to the unusually high correspondence of geometrically implied and perceived slant in this study. Our common experience with pictures and pictorial depth information suggests that we can readily see "depth" from a stimulus we know is two-dimensional. Gibson (1969) has called this taking a "pictorial attitude." Establishment of explicit rectangularity assumptions in the present study was prompted by several studies showing different results with varying instructions (Cappone, 1966; Haber, 1965; McGee, 1963; Power, 1965).

\section{GENERAL CONCLUSIONS}

The Ames window illusion has been a fascinating demonstration, but previous explanations of the effect and its implications have typically been rather vague. Now a direct relation has been established between the perception of static slant and the perception of oscillation in the trapezoidal window illusion. The apparent plane of reversal of a rotating trapezoid is closely related to static slant perceived when the trapezoid is aligned in the frontal plane. Variations in linear perspective and height-width ratio have similar effects on static slant and apparent plane of reversal, although static slant responses show somewhat more regression toward the frontal plane.

A remarkably close correspondence has been demonstrated between perceived and perspective-implied slant. Most previous research in this area has shown a relatively weak relation between projective shapes and perceived slants. Improved stimulus display, a direct response measure, and a geometrically appropriate definition of linear perspective revealed the Ss' ability to respond accurately to linear perspective information. These results give further support to Attneave and Frost's (1969) conclusion that Ss may be rather good perspective geometers.

\section{REFERENCES}

Ames, A. Visual perception and the rotating trapezoidal window. Psychological Monographs, 1951, Whole No, 324.

Attneave, F. Triangles as ambiguous figures. American Journal of Psychology, 1968, 18,643-662.

Attneave, F., \& Frost, R. The determination of perceived tridimensional orientation by minimum criteria. Perception \& Psychophysics, 1969, 6, 391-396.

Börjesson, E. Properties of changing patterns evoking visually perceived oscillation. Perception \& Psychophysics, 1971, 9, 303-308.

Braunstein, M. L., \& Payne, J. W. Perspective and form ratio as determinants of relative slant judgments. Journal of Experimental Psychology, 1969, 81, 584-590.

Burnham, C. A., \& Ono, H. Variables altering perception of the rotating trapezoidal illusion. American Journal of Psychology, $1969,82,86-95$.

Cappone, M. K. The effect of verbal suggestion on the reversal rate of the Ames trapezoidal illusion. Journal of Psychology, $1966,62,211-219$.

Epstein, W., Jansson, G., \& Johansson, G. Perceived angle of oscillatory motion. Perception \& Psychophysics, 1968, 3 , $12-16$.

Flock, H. R., Groves, D., Tenney, J., \& Stephenson, B. Slant judgments of single rectangles at a slant. Psychonomic Science, 1967, 7, 57-58.

Freeman, R. B. Effect of size on visual slant. Journal of Experimental Psychology, 1966, 71, 96-103.

Freeman, R. B., \& Pasnak, R. Perspective determinants of the rotating trapezoid illusion. Journal of Experimental Psychology, 1968, 76, 94-101.

Gibson, J. J., \& Gibson, E. J. Continuous perspective transformations and the perception of rigid motion. Journal of Experimental Psychology, 1957, 54, 129-138.

Gibson, E. J. Principles of perceptual learning and development. New York: Appleton-Century-Crofts, 1969.

Graham, C. H. On some aspects of real and apparent visual movement. Journal of the Optical Society of America, 1963, $63,1019-1025$.

Graham, C. H., \& Gillam, B. J. Occurrence of theoretically correct responses during rotation of the Ames window. Perception \& Psychophysics, 1970, 8, 257-260.

Guenther, W. C. A nalysis of variance, Englewood Cliffs: Prentice-Hall, 1964.

Haber, R. N. Limited modification of the trapezoidal illusion with experience. American Journal of Psychology, 1965, 78, 651-655.

Helmholtz, H. Physiological optics. Vol. 3. New York: Dover, 1962.

Hochberg, J. E. Perception. Englewood Cliffs: Prentice-Hall, 1964. 
Koffka, K. Principles of Gestalt psychology. New York: Harcourt, Brace \& World, 1935 .

McGhee, J. M. The effect of group verbal suggestion and age on the perception of the Ames trapezoidal illusion. Journal of Psychology, 1963, 56, 447-453.

Myers, J. L. Fundamentals of experimental design. Boston: Allyn \& Bacon, 1966.

Pastore, N. Some remarks on the Ames oscillatory effect. Psychological Review, 1952, 59, 319-323.

Power, $R$. P. The effect of instructions on the apparent reversal of rotary motion in depth. Quarterly Journal of Experimental Psychology, 1965, 17, 346-350.

Power, R. P., \& Day, R. H. Constancy and illusion of apparent direction of rotary motion in depth: Tests of a theory.
Perception \& Psychophysics, 1973, 13, 217-223.

Smith, A. H. Relation of slant and shape judgments in reduced viewing. Canadian Journal of Psychology, 1964, 18, 224-234. Wallach, H., \& $O^{\prime}$ Connell, D. N. The kinetic depth effect. Journal of Experimental Psychology, 1953, 45, 205-217.

Wallach, H., O'Connell, D. N., \& Neisser, U. The memory eff ect of visual perception of three dimensional form. Journal of Experimental Psychology, 1953, 45, 360-368.

(Received for publication October 3, 1973; Revision received January 14, 1974.) 